\title{
Expanding the Chemistry Palette for Radiotracer Synthesis
}

\author{
Jennifer Lamb, Faustine d'Orchymont, Rachael Fay, Florian Gribi, Jose Esteban Flores, Melanie \\ Gut, Simon Klingler, Patricia Pires, Jan Bühler, Shamisa Behmaneshfar, Amaury Guillou, Daniel \\ F. Earley, and Jason P. Holland ${ }^{*}$
}

\begin{abstract}
The synthesis, characterisation and application of radiolabelled compounds for use in diagnostic and therapeutic medicine requires a diverse skill set. This article highlights a selection of our ongoing projects that aim to provide new synthetic methods and radiochemical tools for building molecular imaging agents with various radionuclides.
\end{abstract}

Keywords: Antibodies · Bioconjugation - Coordination chemistry - Copper - Density functional theory · Gallium · Graphene · Photochemistry · Positron emission tomography · Protein degradation · Radiochemistry · Zirconium

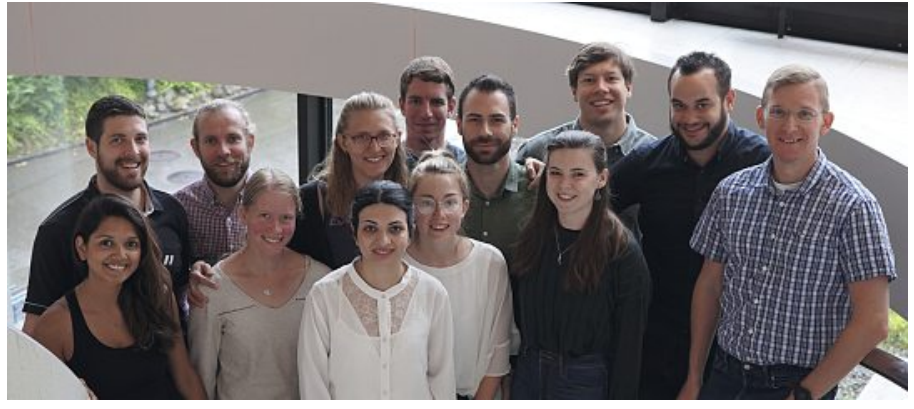

Jason P. Holland is from Yorkshire in the United Kingdom and received a master's degree in Chemistry from the University of York (MChem, 2004) followed by a doctorate from the University of Oxford (DPhil, 2008). He worked at Memorial Sloan-Kettering Cancer Center (2008-2010), ETH Zurich (2010-2012), Harvard Medical School and Massachusetts General (2012-2014), and the University Hospital Freiburg (2015). The Holland group began at the University of Zurich (UZH) in 2016, and our science is funded by the Swiss National Science Foundation, European Research Council, the Krebsliga Schweiz, and the UZH. Research activities focus on advancing radiochemical methods for labelling biological vectors with various radionuclides $\left({ }^{18} \mathrm{~F},{ }^{64} \mathrm{Cu},{ }^{67 / 68} \mathrm{Ga}\right.$, *As, ${ }^{89} \mathrm{Zr}$, ${ }^{99 \mathrm{~m}} \mathrm{Tc},{ }^{111} \mathrm{In},{ }^{177} \mathrm{Lu},{ }^{225} \mathrm{Ac}$ ), chelate design and synthesis, computational chemistry of coordination complexes, and the development of multiple modality imaging agents that target oncogenic pathways in cancer. Photograph (left-to-right): Patricia, Florian, Daniel, Faustine, Melanie, Shamisa, Jan, Jennifer, Jose, Rachael, Simon, Amaury, Jason.

\section{Introduction}

Radiochemistry is one of the most powerful and versatile tools for studying chemical processes at sub-nanomolar levels. Radiolabelled compounds find widespread applications, particularly in Nuclear Medicine as both diagnostic imaging agents and targeted radiotherapeutics. Defining features of working with unstable radionuclides include the half-life and the decay modes. In our group, we primarily work with metal-based radionuclides with half-lives spanning a few minutes to several hours (Fig. 1). Our research focuses on the development of novel radiotracers for use in positron emission tomography (PET) imaging of various cancer biomarkers. Radiotracer development requires an multidisciplinary approach, from organic synthesis, coordination chemistry, inorganic biochemistry, and radiochemistry through to molecular imaging in vivo. In addition, we explore new conjugation methods and radiochemical reactions for introducing radionuclides to a diverse range of biologically active materials from small drug molecules, peptides and proteins, through to nanoparticles. We also perform complete biochemical characterisation of our most promising radiotracers in vitro and imaging studies in tumour-bearing animal models. Collectively, these experiments provide the essential scientific foundation for clinical translation of new radiotracers (Fig. 2).

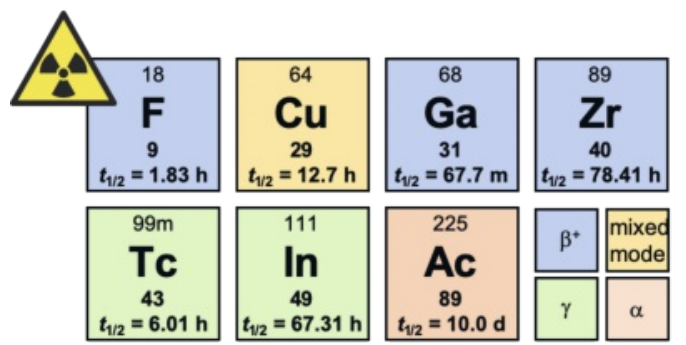

Fig. 1. Radionuclides employed in our group for the development of alternative radiolabelling methods and the synthesis of radiotracers for applications in imaging and therapy.

Radiochemical synthesis lies at the heart of all successful radiopharmaceuticals. Traditional organic and inorganic chemistry offer many elegant reactions that can be adapted for use in radiochemistry but reaction rate is a critical consideration. Our radioactive stock solutions contain, at best, a few nanomoles of the radionuclide and due to the relatively short half-lives, all radiochemical reactions and characterisation should be complete within a few hours from the start of synthesis. Hence, radiochemical kinetics play a central role in determining the success of any labelling process. Our recent concept article provided the first rig- 
Fig. 2. An overview of the main work-flow in our group. Experimental and computational design, synthesis, and complete characterisation is performed in house, with international collaborations facilitating clinical translation.

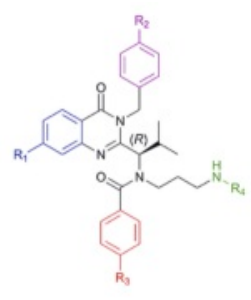

Synthetic chemistry

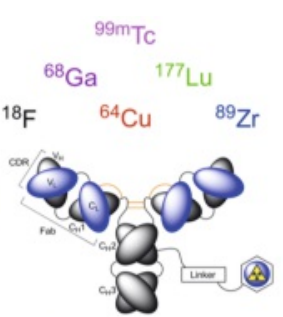

Radiochemistry

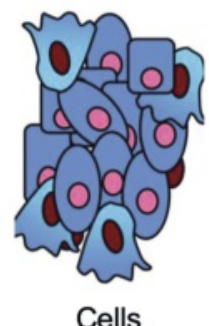

Cells

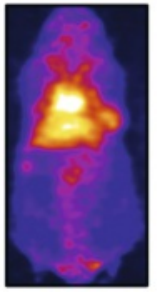

Animals

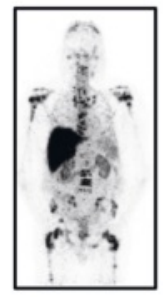

Humans orous formulation of equations and parameters to consider when using chemical kinetics in the context of radiosynthesis. ${ }^{[1]}$ What emerges from the constraints of working with short-lived radionuclides at low concentrations is the notion that faster reactions are required to minimise reaction times and maximise the activity yield. At the same time, when using metal radionuclides, reactions should be compatible with aqueous formulation buffers. In this context, our group is exploring a wide range of alternative methods for constructing multi-functional radiotracers. The following sections provide a brief overview of our ongoing projects that utilise surface-based chemistry, photochemistry, and supramolecular chemistry as novel routes toward radiotracer synthesis. In addition, a core feature of our work with metal radionuclides is the synthesis of new ligands for building metal ion complexes with controlled coordination spheres, high thermodynamic and kinetic stability, and reactive handles for bioconjugation to various cancer-targeting vectors (particularly antibodies). Computational studies with density functional theory (DFT) are used to gain a deeper understanding of the chemistry, electronic structure and spectroscopy of various metal ion complexes, as well as probe reaction mechanisms and streamline our synthetic targets.

\section{Surface-based Radiochemistry and Multi-modality Nanoparticles for Imaging and Therapy}

Nanoparticles are interesting platforms for developing multimodality imaging agents. For example, Bradbury and co-workers reported the clinical translation of ${ }^{124} \mathrm{I}$-radiolabelled ultrasmall inorganic hybrid nanoparticles (C-dots) that offer combined PET and optical imaging options. ${ }^{[2]}$ However, efficient methods for introducing radioactive nuclides without perturbing the physicochemical properties of the particles is one of the main limitations advancing nanomedicines to the clinic. Traditional methods for labelling nanoparticles rely on the use of metal ion binding chelates or prosthetic groups. ${ }^{[3,4]}$ In 2013, pioneering studies from the group of Weibo Cai demonstrated that the intrinsic reactivity of metal oxide nanoparticles can be used to bind radioactive metal ions with high stability and rapid labelling kinetics. ${ }^{[5]}$ In 2015, our group expanded on this initial concept by demonstrating the scope of chelate-free labelling of iron oxide-based nanoparticles using ${ }^{64} \mathrm{Cu},{ }^{89} \mathrm{Zr}$ and ${ }^{111} \mathrm{In}$ radionuclides, as well as various non-radioactive metal ions from across the $p$-, $d$ - and $f$-blocks. ${ }^{[6]}$ Subsequent studies have used the chelate-free approach with a wide range of nanoparticles ${ }^{[7-10]}$ and recent experimental work has supported our initial observations and hypothesis

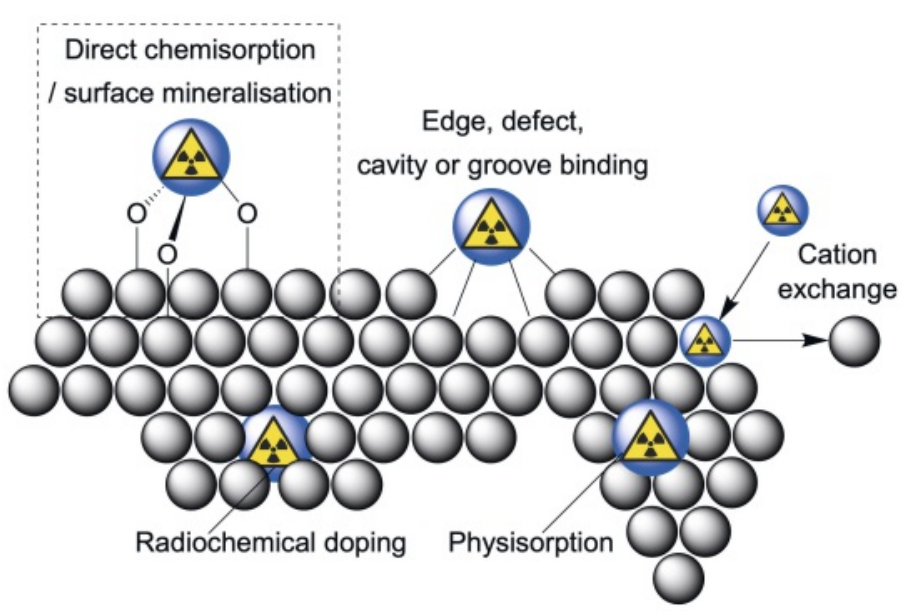

Fig. 3. Illustration of different particle-based binding modes for the chelate-free (intrinsic) radiolabelling of nanoparticles with various radioactive metal ions. ${ }^{[6,12]}$

that the metal ion binding occurs via a mechanism associated with surface (radio)mineralisation (Fig. 3). ${ }^{[11,12]}$

Chelate-free radiolabelling offers a fast and efficient route for introducing different radioactive metal ions onto metal-based nanoparticles. In this context, we continue to explore the mechanism and surface-based reactivity using kinetic studies (Fig. 4), as well as head-to-head comparisons of chelate-free radiochemistry with more traditional labelling methods for use in the development of cancer-targeted iron oxide nanoparticles for multi-modality PET/ magnetic resonance imaging (MRI) of tumours. Experimental work encompasses the synthesis of different nanoparticles with controlled size, composition and coatings (Fig. 5). Nanoparticles are extremely challenging materials to study, but an emerging trend is that surface-reactivity can be exploited for simultaneous radiolabelling and functionalisation with various drug molecules, cancer-specific ligands, and other (optically active) reporter probes. Our general experience is that multi-modality probes can be constructed and show positive tumour-specific binding in vitro, although controlling the pharmacokinetics of the particles in vivo remains a challenge. To this end, we are exploring the use of different coating materials, stabilisers, and protein-based interactions to identify alternative ways of shielding these nanoparticles from non-specific clearance by the reticuloendothelial system.
Fig. 4. Kinetic studies on the intrinsic metal ion radiolabelling of $\mathrm{Fe}_{3} \mathrm{O}_{4}$ nanoparticles by surface (radio)mineralisation. (Left) A schematic of the reaction and various parameters tested in the kinetic studies. (Right) Experimental data showing the percentage radiochemical conversion versus time for ${ }^{68} \mathrm{Ga}$-radiolabelling of $\mathrm{Fe}_{3} \mathrm{O}_{4}$ NPs.
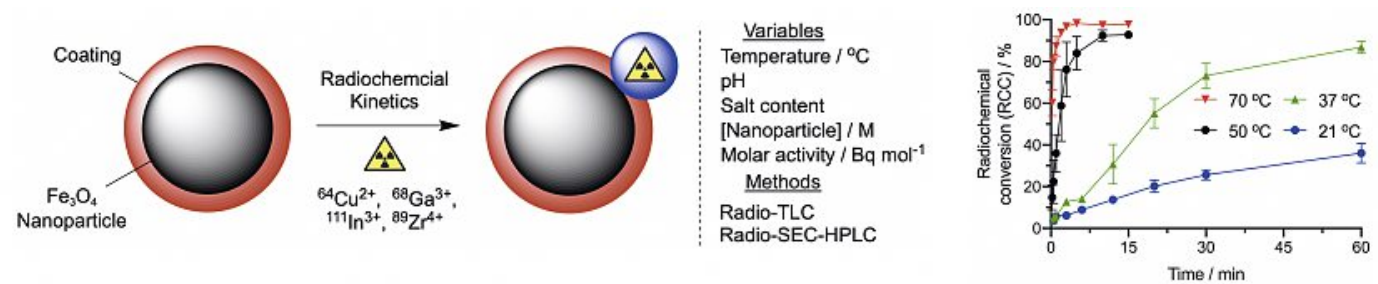

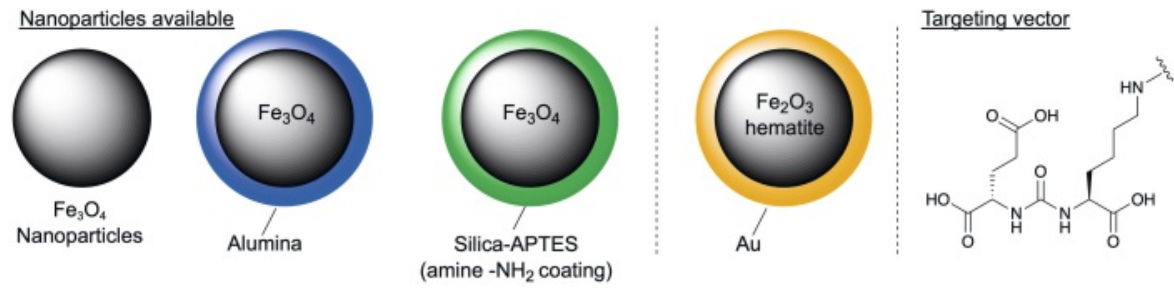

(amine $-\mathrm{NH}_{2}$ coating)

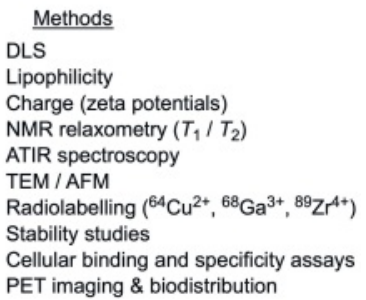

Fig. 5. Synthesis and characterisation of cancer-specific super paramagnetic iron oxide nanoparticles bearing different surface coatings. Surface functionalisation strategies employ several anchoring groups to modify particle loading and stability.

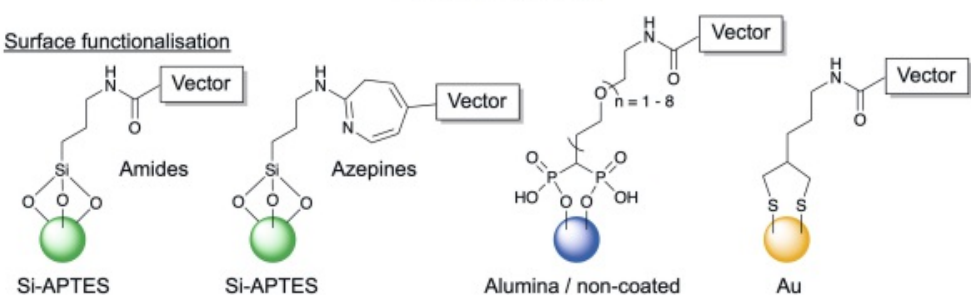

Through an active collaboration with the group of Christoph Salzmann (University College London, UK), we have also expanded our efforts to synthesise multi-modality PET/MRI agents. Here, we combine diagnostic and therapeutic ('theranostic') agents by using graphene nanoflakes (GNFs; Fig. 6) as scaffolds. ${ }^{[13]}$ Initial studies demonstrated that GNFs could be functionalised with multiple ligands at the carboxylated edge via amide bond formation. Subsequent chemical and biochemical assays, including zeta potential and lipophilicity measurements, dynamic light scattering, transmission electron microscopy, radiolabelling, cellular binding and antiproliferation measurements, as well as dynamic PET imaging in vivo are used to investigate the different features of these complex systems. Initial efforts to target cancer biomarkers using small-molecule ligands proved successful in vitro, but again yielded poor pharmacokinetics with rapid excretion via the renal system. Our latest studies are tackling the issue of extending the blood pool circulation time and promoting tumour-specific uptake in vivo by targeting multi-functional GNFs (bearing radionuclides, Gd-based MRI probes and optically active fluorophores) with various antibodies.

\section{New Ligands for Radiometal lons and Applications in Radiotracer Synthesis}

Coordination chemistry is a central tenet of metal-based radiochemistry. Our group is active in this field and we focus primarily on the computational design, synthesis and characterisation of new chelates for complexation of $\mathrm{Cu}^{2+}, \mathrm{Ga}^{3+}, \mathrm{Zr}^{4+}$ and $\mathrm{As}^{3+}$ cations (Fig. 7). Although the overall stability of a radiometal ion complex in vivo is dependent on kinetic and metabolic factors, thermodynamic parameters (as illustrated by the formation

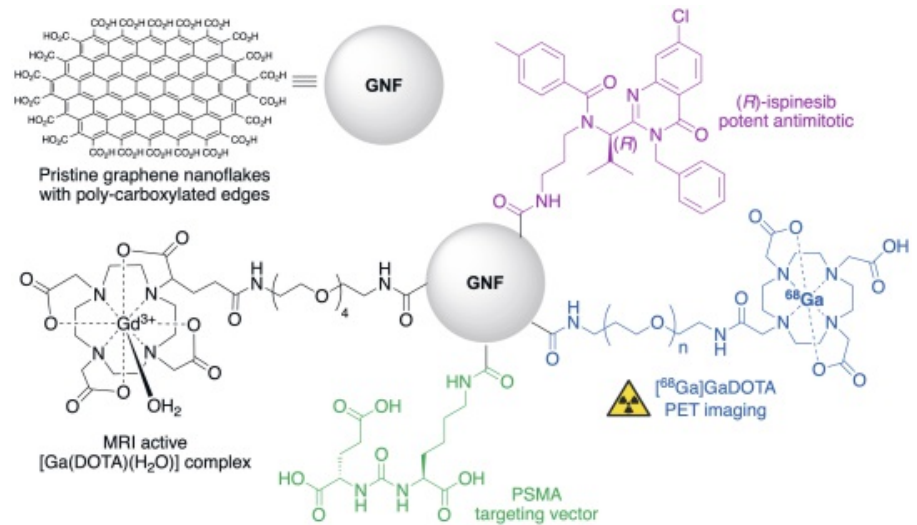

Fig. 6. Schematic structure of a multi-functional graphene nanoflake (GNF) bearing a $\mathrm{Gd}^{3+}$-complex for MRI, a ${ }^{68} \mathrm{Ga}$-complex for PET, a prostate cancer-specific vector targeting PSMA, and a potent antimitotic drug $(R)$-ispinesib capable of inducing mitotic phase arrest by allosteric inhibition of kinesin spindle protein (KSP; hsEg5). constant, $\log \beta$ ) are a primary arbiter in determining the relative complexation power of one ligand versus another. The problem is that classic methods such as potentiometric titration and isothermal calorimetry are often not accessible when working with high-valent (e.g. $\left.\mathrm{Ga}^{3+}, \mathrm{Zr}^{4+}\right)$ metal ion complexes that exhibit formation constants $>30$. Therefore, direct comparison of chelates reported by different research groups presents a challenge. To address this issue, we developed a computational approach for estimating both the relative and absolute thermodynamic stability of different chelates. ${ }^{[14]}$ DFT calculations have proved useful in assisting the design of chelates for ${ }^{89} \mathrm{Zr}^{4+}$ ions which require extremely hard Lewis acid donor sets. Recently our group has shifted direction to explore the coordination chemistry of the extremely soft arsenite $\left(\mathrm{As}^{3+}\right)$ cation. At first sight, arsenic may not appear an obvious choice for developing radiotracers and the element has a long-standing history as a toxic agent - indeed, $\mathrm{As}_{2} \mathrm{O}_{3}$ is an approved therapy for treating leukaemia. At the tracer level, the inherent toxicity of As is not a factor in the dosimetry. Arsenic has at least five radionuclides with potential applications in imaging and therapy: ${ }^{71} \mathrm{As}\left(t_{1 / 2}=65.30 \mathrm{~h}\right),{ }^{72} \mathrm{As}\left(t_{1 / 2}=26.0 \mathrm{~h}\right)$, and ${ }^{74} \mathrm{As}\left(t_{1 / 2}=17.77 \mathrm{~d}\right)$ decay via $\beta^{+}$-emission, whereas ${ }^{74} \mathrm{As}$, ${ }^{76} \mathrm{As}\left(t_{1 / 2}=26.24 \mathrm{~h}\right),{ }^{77} \mathrm{As}\left(t_{1 / 2}=38.79 \mathrm{~h}\right)$ emit $\beta^{-}$-particles that are suitable for radiotherapy. The concept of a matched-pair of radionuclides for applications in imaging and therapy is a driving force for developing As-based radiotracers. Access to new chelates for complexation of $\mathrm{As}^{3+}$ ions would facilitate radiotracer development.

In addition, our synthetic work explores new routes toward multi-dentate $a z a$-macrocycles bearing mixed, N,S,O donor sets for the metal ion selective complexation of $\mathrm{Ga}^{3+}$ and $\mathrm{Cu}^{2+}$ ions. To expand the potential applications of our new chelates for use in radiotracer synthesis and PET imaging, we are synthesising various small-molecule vectors for selective binding to the fibroblast activation protein (FAP) and as potent 'theranostics' for targeted imaging and therapy of prostate cancer via binding to the prostate specific membrane antigen (PSMA) (Fig. 8).
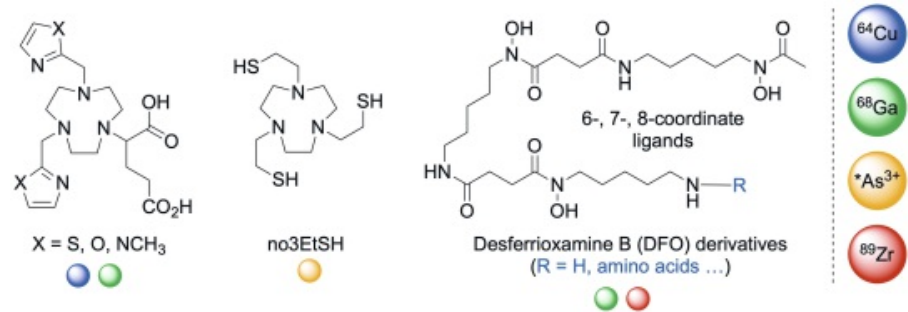

Fig. 7. Representative structures of new chelates for potential complexation of radioactive metal ions including ${ }^{64} \mathrm{Cu}^{2+},{ }^{68} \mathrm{Ga}^{3+},{ }^{89} \mathrm{Zr}^{4+}$, and various radionuclides of ${ }^{*} \mathrm{As}^{3+}$. 
Fig. 8. Small-molecule radiolabelled 'theranostics' that can combine diagnostic imaging with target-specific chemotherapy.

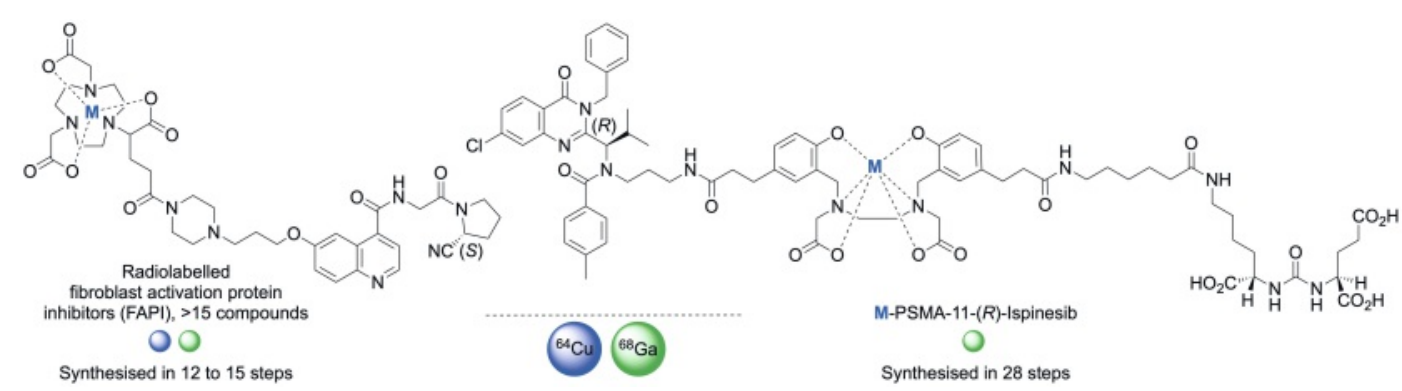

\section{Applications of Photochemistry in Protein-Ligation and Radiotracer Synthesis}

A centrepiece of our recent work is the demonstration that extremely fast, light-induced reactions can be controlled for chemoselective, bimolecular functionalisation of proteins (particularly antibodies [mAbs]).[15-22] The general concept is captured in Fig. 9 and involves the synthesis of photoactivatable metal ion binding chelates, optical probes or potent drug molecules for the one-pot functionalisation of $\mathrm{mAbs}$ at room temperature under mild UV irradiation. As an example, the photolysis of aryl azides with light at 365-395 nm induces efficient activation via the loss of $\mathrm{N}_{2}(\mathrm{~g})$ and yields an extremely reactive (life-time $\sim 1 \mathrm{~ns}$ ) open-shell singlet nitrene. This reaction is well-known and has been utilised in photoaffinity labelling. ${ }^{[23,24]}$ However, in our work, we established conditions whereby the photo-induced products rearrange to a powerful ketenimine electrophile that, remarkably, undergoes kinetically favourable reactions with primary amines. This allows fast, efficient, and chemoselective functionalisation of lysine residues on a protein over background quenching reactions in aerated water. Detailed experimental, spectroscopic, radiochemical and computational studies have allowed us to probe the mechanism and further develop the method. Unlike other standard reagents that are used for protein ligation including activated esters, benzyl-isothiocyanates and maleimides, photochemistry can be used to functionalise $\mathrm{mAbs}$ in formulation buffers (common additives include amino acids, ascorbic acid, sugars and other surfactants). Furthermore, unlike traditional bioconjugation methods which may require multiple steps and extended reaction times, ${ }^{[25]}$ the photochemically induced bioconjugation reactions were found to be first-order in light intensity and are typically complete in $<15$ min. Collectively, these features of highly tolerant chemistry and extremely rapid kinetics at radiotracer concentrations mean that we can now develop fully automated radiosynthesis units for accessing ${ }^{89} \mathrm{Zr}$-mAbs directly from clinical-grade protein samples. Automation would circumvent the need for multiple purification steps, and the expensive synthesis, isolation, characterisation and long-term storage of a functionalised protein-intermediate. Current efforts include work toward the translation of our photora- diosynthesis of ${ }^{89} \mathrm{Zr}$-mAbs for use in the clinic. As our technology develops, we hope that more groups will become interested in exploring the use of photochemistry for making protein conjugates including radiotracers and antibody-drug conjugates (ADCs) with a wider selection of covalent bioconjugate bonds. In the next step, we aim to explore the chemical scope of light-induced reactions by synthesising a range of ligands with photoactivatable moieties that generate (among other species), stabilised singlet nitrenes, carbenes, diradicals, and nitrile imines (Fig. 10).[21] We have also introduced photochemically active fluorophores for tagging proteins (PhotoTags). The synthesis and exploration of the photophysical properties of several different classes of fluorophores are ongoing topics within the group. ${ }^{[26]}$

\section{Supramolecular Chemistry in Radiotracer Design}

In keeping with our theme of developing alternative fast reactions to access radiolabelled constructs with complex architectures, we began exploring the use of supramolecular chemistry in radiotracer design (Fig. 11). Building on a fascinating reaction from Mock et al.,[27] we have adapted Stoddart's 'cooperative capture' concept ${ }^{[28]}$ to synthesise the first radiolabelled rotaxanes. Multi-component reactions involving up to 5 different compounds and 7 functional units in total have been successfully developed to produce several different rotaxanes targeted toward prostate cancer. Molecular features include the use of acyclic and $a z a$-macrocyclic chelates for selective complexation of ${ }^{68} \mathrm{Ga}$ and ${ }^{89} \mathrm{Zr}$ radionuclides, and fluorophores as stoppers on the axel; functionalisation of the cyclodextrin host with cancer-targeting vectors, and metal ion complexes; and stoichiometric control allowing the synthesis of symmetric and asymmetric rotaxanes. Remarkably, this complex, multi-component chemistry is performed in water and we are currently evaluating the properties of several compounds in vitro and in vivo. At this stage, our research remains fundamental but the ultimate goal is to advance this supramolecular technology as a means of fine-tuning the pharmacokinetic profiles of radiotracers via site-selective metabolism and controlled drug release to minimise radiation burden in off-target tissues.

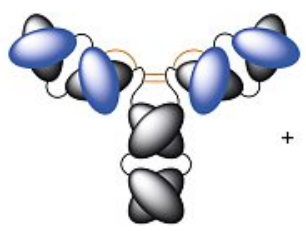

Pre-purified or formulated protein (mAb)

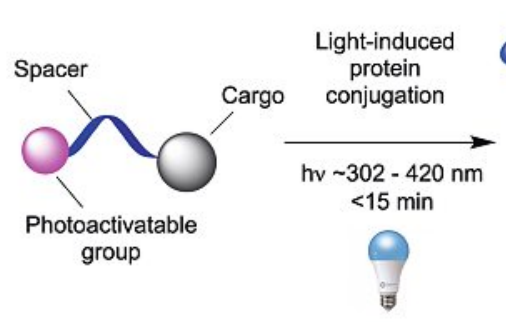

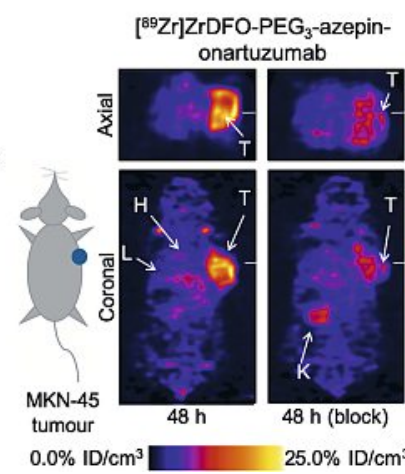

Fig. 9. Schematic illustration of light-induced bimolecular protein-conjugation and photoradiosynthesis of ${ }^{89} \mathrm{Zr}$-mAbs with successful tumour targeting monitored by PET. 

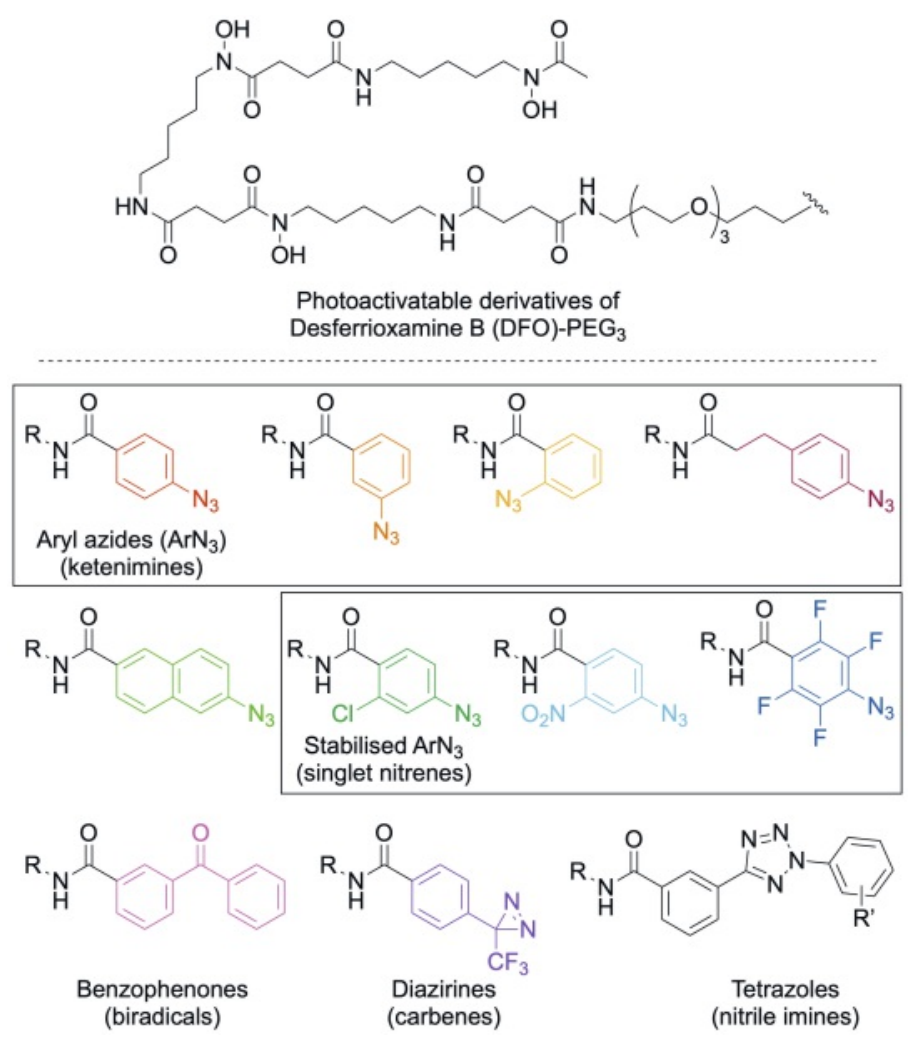

Fig. 10. A selection of photoactivatable DFO derivatives bearing different light-responsive groups.

\section{Transmetallation Chemistry and Fluoride Anion Capture}

Other avenues of ongoing research include the development of transmetallation reactions for radiolabelling ${ }^{64} \mathrm{Cu}$ complexes starting from the non-radioactive $\mathrm{Zn}^{2+}$ precursor species with millisecond kinetics (Fig. 12). This work builds on a concept that was first introduced by the Dilworth group, ${ }^{[29]}$ and our efforts include exploring the chemical scope of transmetallation using different chelates and also different radionuclides. In addition, the aim is to adapt this promising method for the synthesis and validation of small-molecule radiotracers using both homogeneous and heterogeneous phased chemistry.

Fluorine-18 $\left(t_{1 / 2}=109.7 \mathrm{~min}\right)$ is the most common radionuclide used in the development of PET radiotracers. Building on pioneering reports from McBride et al. ${ }^{[30]}$ which demonstrated that fluoride anions can be captured and incorporated into the structure of $\mathrm{Al}^{3+} a z a$-macrocyclic complexes via the formation of a strong Al-F bond, we are exploring the chemical scope of metalfluoride bond formation. Our principle objective is to combine ${ }^{18} \mathrm{~F}$ with high-valent, non-radioactive $\mathrm{Zr}^{4+}$ complexes as a new route toward the synthesis of small-molecule and peptide-based radiotracers via the direct, aqueous-phase capture of ${ }^{18} \mathrm{~F}$ from target solutions (Fig. 13). Successful direct ${ }^{18} \mathrm{~F}$ capture would avoid the time consuming anion trapping and azeotropic drying steps that are a feature of most organic ${ }^{18} \mathrm{~F}$ radiochemistry. Current experiments focus on the synthesis of bifunctional ligands that provide thermodynamically selective and stable complexation of $\mathrm{Zr}^{4+}$ ions, yet feature a vacant site in the first coordination sphere that can accept a fluoride anion selectively in the presence of other

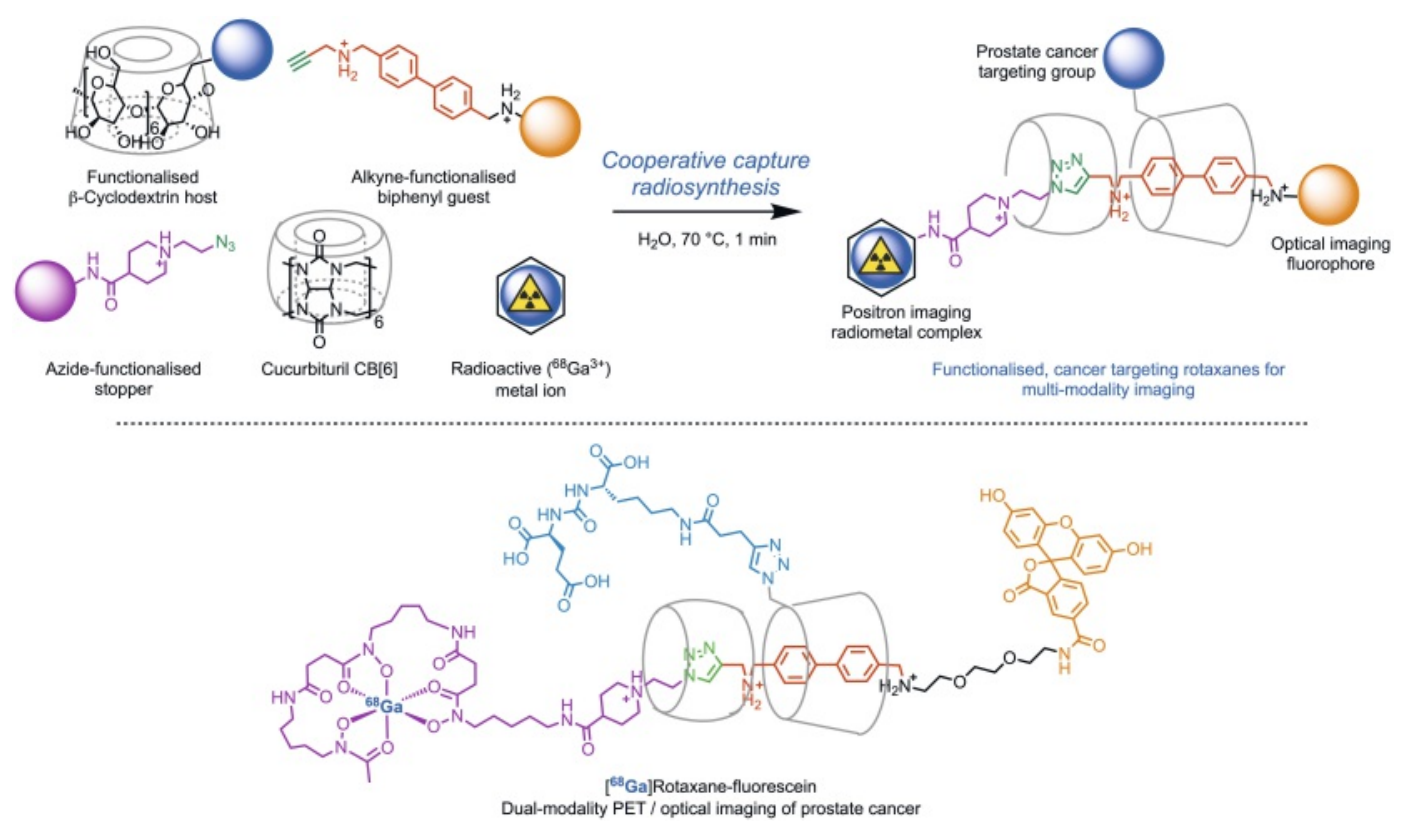

Fig. 11. Supramolecular approaches toward the rapid synthesis of cancer-specific dualmodality imaging agents.
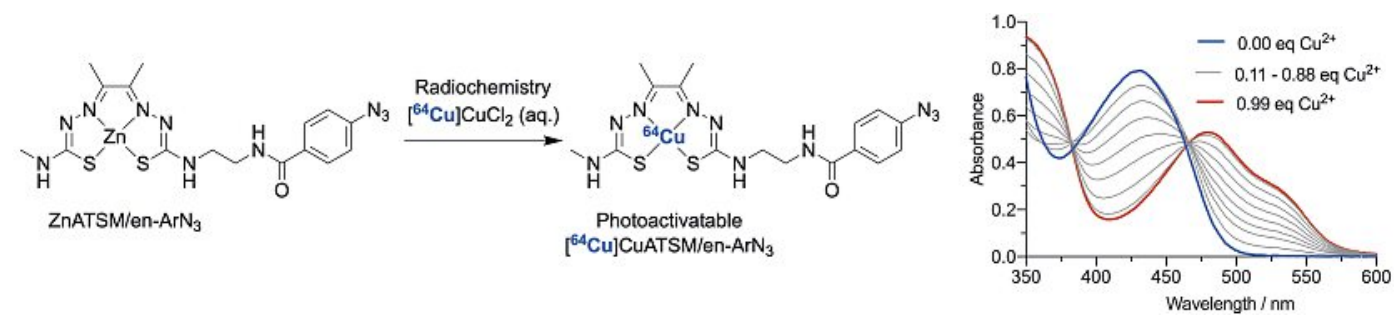

Fig. 12. Rapid ${ }^{64} \mathrm{Cu}$-radiolabelling by transmetallation. 
anions (chloride, phosphates, etc.). In ongoing work, we are using computational studies, synthesis, multinuclear NMR spectroscopy, and radiochemistry to validate the first radiotracers bearing discrete $\mathrm{Zr}-{ }^{18} \mathrm{~F}$ bonds.

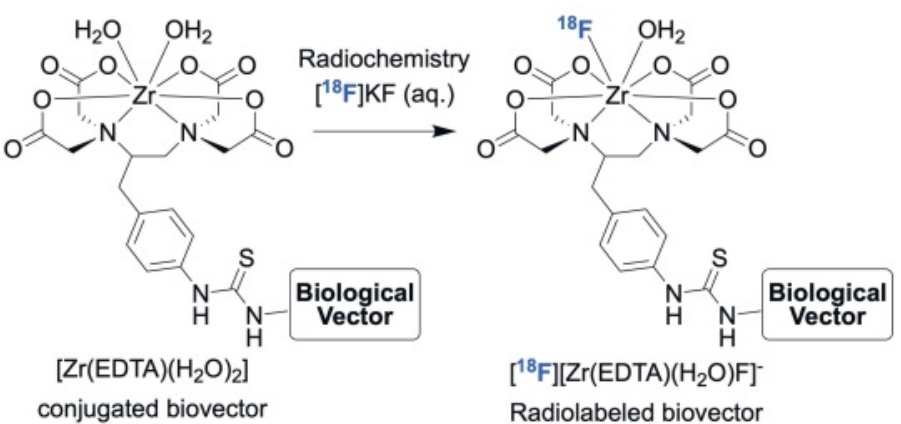

Fig. 13. Aqueous-phase fluoride anion capture by metal ion-fluoride (MF) bond formation.

\section{Companion Diagnostic Imaging of Protein- degradation Therapy in Prostate Cancer}

Our final major topic involves the development of PET radiotracers as companion diagnostic tools to image the action of protein-degrading drugs (Fig. 14). Specifically, our previous work demonstrated that PET imaging can be used to monitor pharmacodynamic changes in downstream protein expression in prostate cancer. Changes in the androgen-receptor (AR) signalling pathway, as well as overexpression and activity of the c-MYC transcription factor were quantified using non-invasive PET coupled with targeted binding to catalytically active 'free' prostate-specific antigen (fPSA), ${ }^{[31]}$ PSMA, ${ }^{[32]}$ and the transferrin receptor (CD71; TfR1). ${ }^{[33,34]}$ Here, our goals are to synthesise a selection of proteolysis targeting chimeric (PROTAC) drugs against AR, and to follow the changes in downstream cellular and tumour biochemistry by using detailed in vitro and ex vivo methods at the protein, mRNA, and metabolomic levels. Ultimately, we aim to use PET imaging as a surrogate for the non-invasive characterisation of drug-target engagement which can then be used for longitudinal observation of changes in therapeutic response, including identifying the onset of chemotherapeutic resistance.

\section{Future Outlook}

Radiochemistry is a fascinating field that requires multidisciplinary skills combined with an ambitious and open mindset. There are research opportunities at all levels from fundamental science to clinic practise that encompass diverse topics such as the invention of new chemical reactions, the experimental and com- putational design of new coordination complexes, and the study of biological processes in vitro and in vivo, all with the ability to deliver essential diagnostic and therapeutic tools for application in Nuclear Medicine. The general philosophy of our research is that the multidisciplinary facets of radiochemistry should not be decoupled but rather, embraced to provide a more complete characterisation and mechanistic understanding of the radiochemical methods and biochemical tool that we develop. Radiochemistry has a bright future ahead.

\section{Acknowledgements}

We thank the Swiss National Science Foundation (SNSF Professorship PP00P2_163683 and PP00P2_190093), the Swiss Cancer League (Krebsliga Schweiz; KLS-4257-08-2017), and the University of Zurich $(\mathrm{UZH})$ for financial support. This project has received funding from the European Union's Horizon 2020 research and innovation programme / from the European Research Council under the Grant Agreement No 676904, ERC-StG-2015, NanoSCAN. We thank the Swiss Government Excellence Scholarship for partial funding of a PhD stipend (FdO; ESKAS-Nr: 2017.0043). We thank all members of the Radiochemistry and Imaging Science group at UZH, past and present, for helpful discussions. In particular, we thank Dr Malay Patra and Larissa Eichenberger for their original work on photoradiochemistry. We also thank our key collaborators Dr Mark Bartholomä (University Hospital Saarbrucken, Germany), Prof. Dr Christoph Salzmann (University College London, United Kingdom), Prof. Dr Rachel Codd (University of Sydney, Australia), Prof. Dr Neil Vasdev (University of Toronto, Canada), Prof. Dr Andreas Plueckthun (University of Zurich), Dr Denis Beckford Vera and Prof. Dr Henry VanBrocklin (University of California San Francisco, United States of America).

Received: August 4, 2020

[1] J. P. Holland, Chem. Eur. J. 2018, 24, 16472

[2] E. Phillips, O. Penate-Medina, P. B. Zanzonico, R. D. Carvajal, P. Mohan, Y. Ye, J. Humm, M. Gönen, H. Kalaigian, H. Schöder, H. W. Strauss, S. M. Larson, U. Wiesner, M. S. Bradbury, Sci. Transl. Med. 2014, 6, 260ra149, https://doi.org:10.1126/scitranslmed.3009524.

[3] S. Goel, F. Chen, E. B. Ehlerding, W. Cai, Small 2014, 10, 3825.

[4] J. Lamb, J. P. Holland, J. Nucl. Med. 2018, 59(3), https://doi.org: 10.2967/jnumed.116.187419.

[5] F. Chen, P. A. Ellison, C. M. Lewis, H. Hong, Y. Zhang, S. Shi, R. Hernandez, M. E. Meyerand, T. E. Barnhart, W. Cai, Angew. Chem. Int. Ed. 2013, 52 , 13319.

[6] E. Boros, A. M. Bowen, L. Josephson, N. Vasdev, J. P. Holland, Chem. Sci. 2015, 6, 225, http://dx.doi.org/10.1039/C4SC02778G.

[7] T. M. Shaffer, M. A. Wall, S. Harmsen, V. A. Longo, C. M. Drain, M. F. Kircher, J. Grimm, Nano Lett. 2015, 15, 864.

[8] T. H. N. Pham, N. A. Lengkeek, I. Greguric, B. J. Kim, P. A. Pellegrini, S A. Bickley, M. R. Tanudji, S. K. Jones, B. S. Hawwett, B. T. T. Pham, Int. J. Nano Med. 2017, 12, 899.

[9] M. A. Wall, T. M. Shaffer, S. Harmsen, D.-F. Tschaharganeh, C.-H. Huang, S. W. Lowe, C. M. Drain, M. F. Kircher, Theranostics 2017, 7, 3068, http://www.thno.org/v07p3068.htm
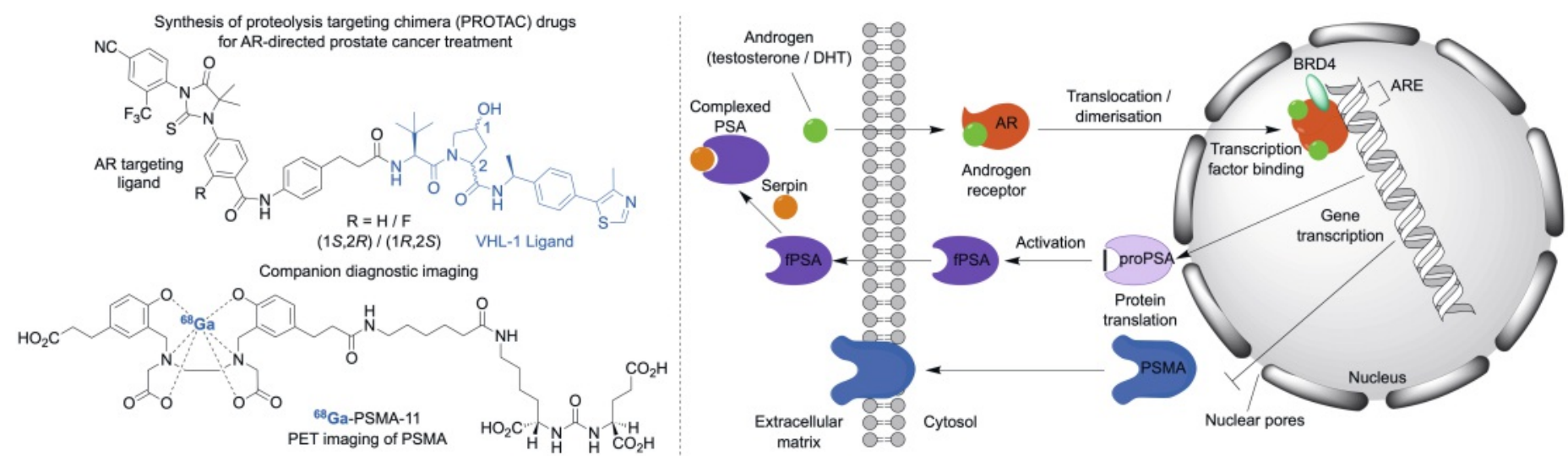

Fig. 14. Synthesis of proteolysis targeting chimeric drugs (PROTACs) and companion diagnostic PET imaging for monitoring the efficacy of protein degradation therapy in androgen-dependent models of prostate cancer. 
[10] M. Radović, M. P. Calatayud, G. F. Goya, M. R. Ibarra, B. Antić, V. Spasojević, N. Nikolić, D. Janković, M. Mirković, S. Vranješ-Đurić, J. Biomed. Mater. Res. A 2015, 103, 126.

[11] P. S. Patrick, L. K. Bogart, T. J. Macdonald, P. Southern, M. J. Powell, M. Zaw-Thin, N. H. Voelcker, I. P. Parkin, Q. A. Pankhurst, M. F. Lythgoe, T. L. Kalber, J. C. Bear, Chem. Sci. 2019, 10, 2592, http://dx.doi.org/10.1039/C8SC04895A

[12] J. Lamb, J. P. Holland, J. Nucl. Med. 2018, 59, 382.

[13] J. Lamb J, E. Fische, M. Rosillo-Lopez, C. G. Salzmann, J. P. Holland, Chem. Sci. 2019, 10, 8880, http://dx.doi.org/10.1039/C9SC03736E

[14] J. P. Holland, Inorg. Chem. 2020, 59, 2070.

[15] M. Patra, L. S. Eichenberger, G. Fischer, J. P. Holland, Angew. Chem. Int. Ed. 2019, 58, 1928.

[16] L. S. Eichenberger, M. Patra, J. P. Holland, Chem. Commun. 2019, 55, 2257.

[17] M. Patra, S. Klingler, L. S. Eichenberger, J. Holland, iScience 2019, 13, 416, https://doi.org/10.1016/j.isci.2019.03.004

[18] R. Fay, M. Gut, J. P. Holland, Bioconjug. Chem. 2019, 30, 1814, http://pubs.acs.org/doi/10.1021/acs.bioconjchem.9b00342

[19] M. Gut, J. P. Holland, Inorg. Chem. 2019, 58, 12302.

[20] S. Klingler, R. Fay, J. P. Holland, S. Klingler, J. Nucl. Med. 2020, 61, 1072.

[21] J. P. Holland, M. Gut, S. Klingler, R. Fay, A. Guillou, Chem. Eur. J. 2020, 26, 33.

[22] A. Guillou, D. F. Earley, J. P. Holland, Chem. Eur. J. 2020, 26, 718, https://doi.org/10.1002/chem.202001755

[23] N. P. Gritsan, M. S. Platz, Chem. Rev. 2006, 106, 3844.

[24] W. T. Borden, N. P. Gritsan, C. M. Hadad, W. L. Karney, C. R. Kemnitz, M. S. Platz, Acc. Chem. Res. 2000, 33, 765.

[25] P. Adumeau, D. Vivier, S. K. Sharma, J. Wang, T. Zhang, A. Chen, B. J. Agnew, B. M. Zeglis, Mol. Pharm. 2018, 15, 892.

[26] R. Fay, A. Linden, J. P. Holland, Org. Lett. 2020, 22, 3499.
[27] W. L. Mock, N. Y. Shih, J. Org. Chem. 1983, 48, 3618.

[28] X. Hou, C. Ke, J. F. Stoddart, Chem. Soc. Rev. 2016, 45, 3766.

[29] H. M. Betts, P. J. Barnard, S. R. Bayly, J. R. Dilworth, A. D. Gee, J. P. Holland, Angew. Chem. Int. Ed. 2008, 47, 8416.

[30] W. J. McBride, R. M. Sharkey, H. Karacay, C. A. D’Souza, E. A. Rossi, P. Laverman, C.-H. Chang, O. C. Boermann, D. M. Goldenberg, J. Nucl. Med. 2009, 50, 991,

http://jnm.snmjournals.org/cgi/doi/10.2967/jnumed.108.060418

[31] D. Ulmert, M. J. Evans, J. P. Holland, S. L. Rice, J. Wongvipat, K. Pettersson, P.-A. Abrahamsson, P. T. Scardino, S. M. Larson, H. Lilja, J. S. Lewis, C. L. Sawyers, Cancer Discov. 2012, 2, 320.

[32] M. J. Evans, P. M. Smith-jones, J. Wongvipat, V. Navarro, S. Kim, N. H. Bander, Proc. Natl. Acad. Sci. USA 2011, 108, 9578.

[33] J. P. Holland, M. J. Evans, S. L. Rice, J. Wongvipat, C. L. Sawyers, J. S. Lewis, Nat. Med. 2012, 18, 1586, http://www.nature.com/doifinder/10.1038/nm.2935

[34] M. J. Evans, J. P. Holland, S. L. Rice, M. G. Doran, S. M. Cheal, C. Campos, S. D. Carlin, I. K. Mellinghoff, C. L. Sawyers, J. S. Lewis, J. Nucl. Med. 2013, 54, 90 .

\section{License and Terms}

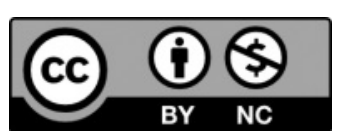

This is an Open Access article under the terms of the Creative Commons Attribution License CC BY_NC 4.0. The material may not be used for commercial purposes.

The license is subject to the CHIMIA terms and conditions: (http:// chimia.ch/component/sppagebuilder/?view=page\&id=12).

The definitive version of this article is the electronic one that can be found at https://doi.org/10.2533/chimia.2020.946 